\title{
ON GENERALIZED MOMENT MATRICES
}

\author{
RUIMING ZHANG
}

(Communicated by Sergei K. Suslov)

\begin{abstract}
In this work we provide a method to compute the determinants and inverses of the generalized moment matrices. Using this method we also give lower bounds for the smallest eigenvalue of such matrices.
\end{abstract}

\section{INTRODUCTION}

It is well-known that Gram matrices generalize moment matrices and that the generalized moment matrices extend the classical Hilbert matrices, [6] thus it is natural to consider the inverses and determinants of generalized moment matrices. The location of the smallest eigenvalue of such matrices is also a very interesting problem; for example, see [2]. In this note we will demonstrate that, just as in the case for Hilbert matrices, we can find closed formulas for determinants and inverses for generalized moment matrices systematically. Furthermore, we provide lower bounds for the smallest eigenvalue of the matrices.

Given a probability measure $d \mu(x)$ on $\mathbb{R}$ such that $\int_{\mathbb{R}} x^{2 n} d \mu(x)<\infty$ for all $n \in \mathbb{R}$, we define the inner product for $d \mu(x)$ square integrable functions $f(x)$ and $g(x)$ by

$$
(f, g)=\int_{-\infty}^{\infty} f(x) g(x) d \mu(x) .
$$

For each $n \in \mathbb{N} \cup\{0\}$, let $G_{n}=\left(m_{j, k}\right)_{j, k=0}^{n}$ with $m_{j, k}=\left(u_{j}, u_{k}\right)$ for $j, k=0,1, \ldots, n$, where $\left\{u_{k}(x)\right\}_{k=0}^{\infty}$ is a sequence of polynomials with $u_{0}(x)=1$ such that for each $k, u_{k}(x)$ is of degree exactly $k$. Then there is an essentially unique orthonormal system $\left\{p_{k}(x)\right\}_{k=0}^{\infty}$ [1, 3, 5],

$$
p_{n}(x)=\frac{1}{\sqrt{\operatorname{det} G_{n} \operatorname{det} G_{n-1}}} \operatorname{det}\left(\begin{array}{ccccc}
m_{0,0} & m_{0,1} & m_{0,2} & \ldots & m_{0, n} \\
m_{1,0} & m_{1,1} & m_{1,2} & \ldots & m_{1, n} \\
\vdots & \vdots & \vdots & \ddots & \vdots \\
m_{n-1,0} & m_{n-1,1} & m_{n-1,2} & \ldots & m_{n-1, n} \\
u_{0}(x) & u_{1}(x) & u_{2}(x) & \ldots & u_{n}(x)
\end{array}\right)
$$

with positive leading coefficient in $u_{n}(x)$. Clearly we have $p_{n}(x)=\sum_{j=0}^{n} a_{n, j} u_{j}(x)$ for some real numbers $a_{j, k}$ for $j, k=0,1, \ldots, n$ and $a_{j, k}=0$ for $k>j$. We state our main results as a theorem:

Received by the editors October 28, 2011.

2000 Mathematics Subject Classification. Primary 15A09; Secondary 33D45.

Key words and phrases. Orthogonal polynomials, generalized moment matrices, determinants, inverse matrices, smallest eigenvalue.

This work was partially supported by Northwest A\&F University. 
Theorem 1. For each nonnegative integer $n$, let $G_{n}=\left(m_{j, k}\right)_{j, k=0}^{n}$ and $A_{n}=$ $\left(a_{j, k}\right)_{j, k=0}^{n}$. Then

$$
\operatorname{det} G_{n}=\prod_{j=0}^{n} a_{j, j}^{-2}, \quad G_{n}^{-1}=A_{n}^{T} A_{n} .
$$

Furthermore, let $\lambda_{s}(n)$ be the smallest eigenvalue of $G_{n}$. Then

$$
\lambda_{s}(n) \geq \frac{1}{\sum_{\ell=0}^{n}\left\{\sum_{j=0}^{\ell}\left|a_{\ell, j}\right|\right\}^{2}} .
$$

Remark 2 . In the case $u_{j}(x)=x^{j}, j=0,1, \ldots$, for the most of orthogonal polynomials we can find a $z_{0}$ with $\left|z_{0}\right|=1$ such that for each $n$, all $a_{n, j} z_{0}^{j}, j=0,1, \ldots, n$, have the same sign. Then we have $\sum_{j=0}^{\ell}\left|a_{\ell, j}\right|=\left|p_{\ell}\left(z_{0}\right)\right|$ and (1.4) becomes

$$
\lambda_{s}(n) \geq \frac{1}{\sum_{\ell=0}^{n}\left\{p_{\ell}\left(z_{0}\right)\right\}^{2}},
$$

which can be written as

$$
\lambda_{s}(n) \geq \frac{a_{n+1, n+1}}{a_{n, n}\left\{p_{n+1}^{\prime}\left(z_{0}\right) p_{n}\left(z_{0}\right)-p_{n}^{\prime}\left(z_{0}\right) p_{n+1}\left(z_{0}\right)\right\}}
$$

by the Christoffel-Darboux formula. From the theory of orthogonal polynomials it is known that the right hand side of (1.5) has a positive limit for an indeterminate moment problem, whereas it has 0 as the limit for a determinant moment problem [2, 3].

\section{Proofs}

For each $n \in \mathbb{N} \cup\{0\}$ and $x_{i}, a_{i, j} \in \mathbb{C}$ for $i, j=0,1, \ldots, n$, we define

$$
A=\left(\begin{array}{cccc}
a_{0,0} & a_{0,1} & \ldots & a_{0, n} \\
a_{1,0} & a_{1,1} & \ldots & a_{1, n} \\
\vdots & \vdots & \ddots & \vdots \\
a_{n, 0} & a_{n, 1} & \ldots & a_{n, n}
\end{array}\right), \quad x=\left(\begin{array}{c}
x_{0} \\
x_{1} \\
\vdots \\
x_{n}
\end{array}\right)
$$

and

$$
\|A\|=\sup _{x \neq 0} \frac{\|A x\|}{\|x\|}, \quad\|x\|=\sqrt{\sum_{i=0}^{b}\left|x_{i}\right|^{2}} .
$$

Lemma 3. For each $n \in \mathbb{N} \cup\{0\}$ we have $\|A\| \leq C$, where

$$
C=\sqrt{\left\{\max _{j} \sum_{i=0}^{n}\left|a_{i, j}\right|\right\} \cdot\left\{\max _{i} \sum_{j=0}^{n}\left|a_{i, j}\right|\right\}} .
$$

In particular, if $A$ is positive definite, then

$$
\lambda=\|A\| \leq \max _{i} \sum_{j=0}^{n}\left|a_{i, j}\right|,
$$

where $\lambda$ is the largest eigenvalue of $A$. 
Proof. For any

$$
x=\left(\begin{array}{c}
x_{0} \\
x_{1} \\
\vdots \\
x_{n}
\end{array}\right), \quad y=\left(\begin{array}{c}
y_{0} \\
y_{1} \\
\vdots \\
y_{n}
\end{array}\right)
$$

for $x_{i}, y_{i} \in \mathbb{C}, i=0,1, \ldots n$, then

$$
\begin{aligned}
|(A x, y)| & =\left|\sum_{i=0}^{n} \sum_{j=0}^{n} a_{i, j} x_{j} \overline{y_{i}}\right| \\
& \leq \sum_{i=0}^{n} \sum_{j=0}^{n}\left\{\sqrt{\left|a_{i, j}\right|}\left|x_{j}\right|\right\}\left\{\sqrt{\left|a_{i, j}\right|}\left|y_{i}\right|\right\} \\
& \leq \sqrt{\sum_{i=0}^{n} \sum_{j=0}^{n}\left|a_{i, j}\right|\left|x_{j}\right|^{2}} \cdot \sqrt{\sum_{i=0}^{n} \sum_{j=0}^{n}\left|a_{i, j}\right|\left|y_{i}\right|^{2}} \\
& =\sqrt{\left\{\max _{j} \sum_{i=0}^{n}\left|a_{i, j}\right|\right\} \sum_{j=0}^{n}\left|x_{j}\right|^{2}} \cdot \sqrt{\left\{\max _{i} \sum_{j=0}^{n}\left|a_{i, j}\right|\right\} \sum_{i=0}^{n}\left|y_{i}\right|^{2}} \\
& =C\|x\| \cdot\|y\| .
\end{aligned}
$$

For any nonzero vector $x$, let $y=A x$ to get $\|A x\|^{2} \leq C\|x\| \cdot\|A x\|$, which implies $\|A\| \leq C$. Let $A$ be a positive definite matrix and $\lambda$ be its largest eigenvalue; then $\lambda=\|A\|$ by the spectral theorem. Since $a_{i, j}=a_{j, i}$ for $i, j=0,1, \ldots, n$, then $C=\max _{j} \sum_{i=0}^{n}\left|a_{i, j}\right|$, and consequently we have already proved (2.4).

2.1. Proof of Theorem 1. From (1.2) and $p_{n}(x)=\sum_{j=0}^{n} a_{n, j} u_{j}(x)$ it is clear that

$$
a_{n, n}=\sqrt{\frac{\operatorname{det} G_{n-1}}{\operatorname{det} G_{n}}}, \quad \operatorname{det} G_{n}=\prod_{j=0}^{n} a_{j, j}^{-2} .
$$

Since both $\left\{p_{k}(x)\right\}_{k=0}^{n}$ and $\left\{u_{k}(x)\right\}_{k=0}^{n}$ form a basis for all the polynomials of degree $n, A_{n}$ must be invertible for each $n \in \mathbb{N} \cup\{0\}$. We denote $A_{n}^{-1}=\left(s_{j, k}\right)_{j, k=0}^{n}$; then $u_{j}(x)=\sum_{\ell=0}^{n} s_{j, \ell} p_{\ell}(x)$ for each $j=0,1, \ldots n$. Clearly, $s_{j, \ell}=0$ for $\ell>j$. Thus,

$$
m_{j, k}=\left(u_{j}(x), u_{k}(x)\right)=\sum_{m=0}^{n} s_{j, m} s_{k, m}
$$

for $j, k=0,1, \ldots, n$, which is

$$
G_{n}=A_{n}^{-1}\left(A_{n}^{-1}\right)^{T}=A_{n}^{-1}\left(A_{n}^{T}\right)^{-1} .
$$

Then $G_{n}^{-1}=A_{n}^{T} A_{n}$. Since $G_{n}$ is positive definite, its smallest eigenvalue is positive. Let $\lambda_{s}(n)>0$ be the smallest eigenvalue; then $\frac{1}{\lambda_{s}(n)}$ is the largest eigenvalue of $G_{n}^{-1}$ by the spectral theorem. Let $G_{n}^{-1}=\left(\gamma_{j, k}\right)_{j, k=0}^{n}$; then

$$
\gamma_{j, k}=\sum_{\ell=0}^{n} a_{\ell, j} a_{\ell, k}, \quad j, k=0,1, \ldots, n .
$$


Applying Lemma 3 we obtain

$$
\begin{aligned}
\frac{1}{\lambda_{s}(n)} & \leq \max _{j} \sum_{k=0}^{n}\left|\gamma_{j, k}\right| \leq \max _{j} \sum_{k=0}^{n} \sum_{\ell=0}^{n}\left|a_{\ell, j}\right|\left|a_{\ell, k}\right| \\
& \leq \sum_{\ell=0}^{n}\left\{\sum_{j=0}^{n}\left|a_{\ell, j}\right|\right\}\left\{\sum_{k=0}^{n}\left|a_{\ell, k}\right|\right\}=\sum_{\ell=0}^{n}\left\{\sum_{j=0}^{n}\left|a_{\ell, j}\right|\right\}^{2},
\end{aligned}
$$

which gives (1.4).

\section{EXAMPLES}

3.1. Matrices related to Laguerre polynomials. The normalized Laguerre polynomials $\left\{\ell_{n}^{(\alpha)}(x)\right\}_{n=0}^{\infty}$ are defined by [1, 3, 4, 5]

$$
\ell_{n}^{(\alpha)}(x)=(-1)^{n} \sqrt{\frac{n !}{(\alpha+1)_{n}}} L_{n}^{\alpha}(x)=\sqrt{\frac{(\alpha+1)_{n}}{n !}} \sum_{k=0}^{n} \frac{(-1)^{n}(-n)_{k} x^{k}}{(\alpha+1)_{k} k !}
$$

for $n \geq 0$, and we assume that $\ell_{-1}^{(\alpha)}(x)=0$, where

$$
(a)_{n}=\frac{\Gamma(a+n)}{\Gamma(a)}, \quad \frac{1}{\Gamma(a)}=a \prod_{j=1}^{\infty}\left(1+\frac{a}{j}\right)\left(1+\frac{1}{j}\right)^{-a}
$$

for $a \in \mathbb{C}$ and $n \in \mathbb{Z}$. Under the condition $\alpha>-1$, they satisfy

$$
\int_{0}^{\infty} \ell_{n}^{(\alpha)}(x) \ell_{m}^{(\alpha)}(x) \frac{x^{\alpha} e^{-x}}{\Gamma(\alpha+1)} d x=\delta_{m n}, \quad m, n=0,1, \ldots,
$$

and the $n$-th moment of the measure is

$$
m_{n}=\frac{\int_{0}^{\infty} x^{\alpha+n} e^{-x} d x}{\Gamma(\alpha+1)}=(\alpha+1)_{n}
$$

for $n=0,1, \ldots$ From (3.1) we obtain

$$
a_{n, k}=\sqrt{\frac{(\alpha+1)_{n}}{n !}} \frac{(-n)_{k}(-1)^{n}}{(\alpha+1)_{k} k !}
$$

for $n, k=0,1, \ldots$ by taking $\mu_{j}(x)=x^{j}$ for $j=0,1, \ldots$ Applying Theorem 1 we get

$$
\operatorname{det}\left((\alpha+1)_{j+k}\right)_{0 \leq j, k \leq n}=\prod_{k=0}^{n}\left\{k !(\alpha+1)_{k}\right\}
$$

and the inverse matrix

$$
\left((\alpha+1)_{j+k}\right)_{0 \leq j, k \leq n}^{-1}=\left(\sum_{\ell=0}^{n} \frac{(\alpha+1)_{\ell}(-\ell)_{j}(-\ell)_{k}}{\ell !(\alpha+1)_{j}(\alpha+1)_{k} j ! k !}\right)_{j, k=0}^{n} .
$$


The smallest eigenvalue of the matrix $\left((\alpha+1)_{j+k}\right)_{0 \leq j, k \leq n}$ is given by

$$
\begin{aligned}
\lambda_{s}(n) \geq\left\{\sum_{\ell=0}^{n} \frac{\ell !}{(\alpha+1)_{\ell}} L_{\ell}^{(\alpha)}(-1)^{2}\right\}^{-1} \\
=\frac{(\alpha+1)_{n}}{(n+1) !} \frac{1}{L_{n}^{(\alpha+1)}(-1) L_{n}^{(\alpha)}(-1)-L_{n+1}^{(\alpha)}(-1) L_{n-1}^{(\alpha+1)}(-1)},
\end{aligned}
$$

where we have applied the formula

$$
\frac{d}{d x} L_{n}^{(\alpha)}(x)=-L_{n-1}^{(\alpha+1)}(x) .
$$

From the Perron formula [3, 5,

$$
L_{n}^{(\alpha)}(x)=\frac{e^{x / 2}}{2 \pi}(-x)^{-\alpha / 2-1 / 4} n^{\alpha / 2-1 / 4} \exp \left\{2(-n x)^{1 / 2}\right\}\left\{1+\mathcal{O}\left(\frac{1}{n^{1 / 2}}\right)\right\}
$$

for $x \in \mathbb{C} \backslash(0, \infty)$, we get

$$
L_{n}^{(\alpha+1)}(-1) L_{n}^{(\alpha)}(-1)-L_{n+1}^{(\alpha)}(-1) L_{n-1}^{(\alpha+1)}(-1)=\frac{n^{\alpha-1} \exp \left(4 n^{1 / 2}\right)}{8 \pi e}\left\{1+\mathcal{O}\left(\frac{1}{n^{1 / 2}}\right)\right\}
$$

as $n \rightarrow \infty$. This together with

$$
\frac{\Gamma(n+\alpha)}{\Gamma(n+\beta)}=n^{\alpha-\beta}\left\{1+\mathcal{O}\left(\frac{1}{n}\right)\right\}
$$

gives

$$
\left\{\sum_{\ell=0}^{n} \frac{\ell !}{(\alpha+1)_{\ell}} L_{\ell}^{(\alpha)}(-1)^{2}\right\}^{-1}=\frac{8 \pi e}{\Gamma(\alpha+1)} \exp \left(-4 n^{1 / 2}\right)\left\{1+\mathcal{O}\left(\frac{1}{n^{1 / 2}}\right)\right\}
$$

as $n \rightarrow \infty$.

3.2. Matrices related to the $q$-Laguerre polynomials. The normalized $q$ Laguerre polynomials $\left\{\ell_{n}^{(\alpha)}(x ; q)\right\}_{n=0}^{\infty}$ are defined by [1, 3, 4]

$$
\begin{aligned}
\ell_{n}^{(\alpha)}(x ; q) & =(-1)^{n} \sqrt{\frac{(q ; q)_{n} q^{n}}{\left(q^{\alpha+1} ; q\right)_{n}}} L_{n}^{(\alpha)}(x ; q) \\
& =\sqrt{\frac{\left(q^{\alpha+1} ; q\right)_{n} q^{n}}{(q ; q)_{n}}} \sum_{k=0}^{n} \frac{\left.\left(q^{-n} ; q\right)_{k} q^{(k+1}\right)_{2} q^{(\alpha+n) k} x^{k}}{(-1)^{n}(q ; q)_{k}\left(q^{\alpha+1} ; q\right)_{k}}
\end{aligned}
$$

for $n \geq 0$, and we assume that $\ell_{-1}^{(\alpha)}(x ; q)=0$, where

$$
(a ; q)_{\infty}=\prod_{k=0}^{\infty}\left(1-a q^{k}\right),(a ; q)_{n}=\frac{(a ; q)_{\infty}}{\left(a q^{n} ; q\right)_{\infty}}
$$

and

$$
\left(a_{1}, a_{2}, \ldots, a_{m} ; q\right)_{n}=\prod_{j=1}^{m}\left(a_{j} ; q\right)_{n}
$$


for $z \in \mathbb{C}, q \in(0,1), n \in \mathbb{Z}$ and $m \in \mathbb{N}$. The moment problem of the $q$-Laguerre polynomials is indeterminate, and one of the orthogonalities is 3 , 4]

$$
\int_{0}^{\infty} \ell_{n}^{(\alpha)}(x ; q) \ell_{m}^{(\alpha)}(x ; q) w(x) d x=\delta_{m, n}
$$

for $m, n \geq 0$ with

$$
w(x)=-\frac{\sin \pi \alpha}{\pi} \frac{(q ; q)_{\infty} x^{\alpha}}{\left(q^{-\alpha},-x ; q\right)_{\infty}} .
$$

Also, the $n$-th moment is

$$
\mu_{n}(\alpha)=\left(q^{\alpha+1} ; q\right)_{n} q^{-\alpha n-\left(\begin{array}{c}
n+1 \\
2
\end{array}\right)},
$$

and the orthonormal system is given by

$$
\ell_{n}^{(\alpha)}(x ; q)=\sqrt{\frac{\left(q^{\alpha+1} ; q\right)_{n} q^{n}}{(q ; q)_{n}}} \sum_{k=0}^{n} \frac{\left(q^{-n} ; q\right)_{k} q^{\left(\begin{array}{c}
k+1 \\
2
\end{array}\right)} q^{(\alpha+n) k} x^{k}}{(-1)^{n}(q ; q)_{k}\left(q^{\alpha+1} ; q\right)_{k}} .
$$

From (3.14) we get

$$
a_{n, k}=\sqrt{\frac{\left(q^{\alpha+1} ; q\right)_{n} q^{n}}{(q ; q)_{n}} \frac{\left(q^{-n} ; q\right)_{k} q^{\left(\begin{array}{c}
k+1 \\
2
\end{array}\right)} q^{(\alpha+n) k}}{(-1)^{n}(q ; q)_{k}\left(q^{\alpha+1} ; q\right)_{k}}}
$$

by taking $\mu_{j}(x)=x^{j}$ for $j=0,1, \ldots$ Applying Theorem 1 we prove that the matrix

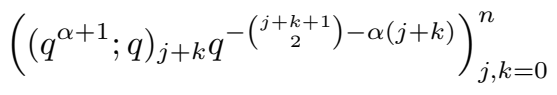

has inverse $\left(\gamma_{j, k}\right)_{j, k=0}^{n}$ with

$$
\gamma_{j, k}=\sum_{m=0}^{n} \frac{\left(q^{\alpha+1} ; q\right)_{m} q^{m(j+k+1)}\left(q^{-m} ; q\right)_{j}\left(q^{-m} ; q\right)_{k} q^{\alpha(j+k)+\left(\begin{array}{c}
j+1 \\
2
\end{array}\right)+\left(\begin{array}{c}
k+1 \\
2
\end{array}\right)}}{(q ; q)_{m}(q ; q)_{j}(q ; q)_{k}\left(q^{\alpha+1} ; q\right)_{j}\left(q^{\alpha+1} ; q\right)_{k}}
$$

and that

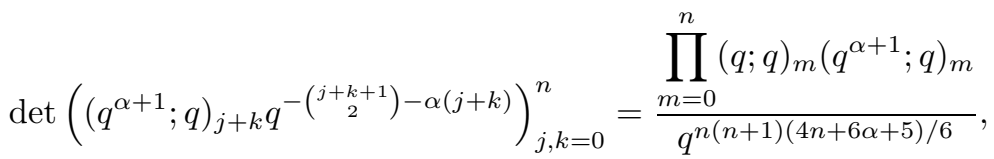

a lower bound for the smallest eigenvalue, is given by

$$
\left(\sum_{m=0}^{n}\left|\ell_{m}^{(\alpha)}(-1 ; q)\right|^{2}\right)^{-1}
$$

But

$$
\begin{aligned}
\ell_{m}^{(\alpha)}(-1 ; q) & =\sqrt{\frac{\left(q^{\alpha+1} ; q\right)_{m} q^{m}}{(q ; q)_{m}} \sum_{k=0}^{m} \frac{\left(q^{-m} ; q\right)_{k} q^{\left(\begin{array}{c}
k \\
2
\end{array}\right)}\left(-q^{m+\alpha+1}\right)^{k}}{(-1)^{m}(q ; q)_{k}\left(q^{\alpha+1} ; q\right)_{k}}} \\
& =(-1)^{m} \sqrt{\frac{\left(q^{\alpha+1} ; q\right)_{m} q^{m}}{(q ; q)_{m}}} \frac{\left(q^{m+\alpha+1} ; q\right)_{\infty}}{\left(q^{\alpha+1} ; q\right)_{\infty}}=(-1)^{m} \sqrt{\frac{q^{m}}{\left(q, q^{\alpha+1} ; q\right)_{m}}}
\end{aligned}
$$


thus this lower bound for the smallest eigenvalue is

$$
\left(\sum_{m=0}^{n} \frac{q^{m}}{\left(q, q^{\alpha+1} ; q\right)_{m}}\right)^{-1}
$$

It is clear that

$$
\begin{aligned}
& \sum_{m=0}^{n} \frac{q^{m}}{\left(q, q^{\alpha+1} ; q\right)_{m}} \leq \sum_{m=0}^{\infty} \frac{q^{m}}{\left(q, q^{\alpha+1} ; q\right)_{m}} \\
& \leq \frac{1}{\left(q^{\alpha+1} ; q\right)_{\infty}} \sum_{m=0}^{\infty} \frac{q^{m}}{(q ; q)_{m}}=\frac{1}{\left(q, q^{\alpha+1} ; q\right)_{\infty}}
\end{aligned}
$$

hence

$$
\lambda_{s}(n) \geq\left(\sum_{m=0}^{\infty} \frac{q^{m}}{\left(q, q^{\alpha+1} ; q\right)_{m}}\right)^{-1} \geq\left(q, q^{\alpha+1} ; q\right)_{\infty}
$$

for all $n \in \mathbb{N}$.

\section{REFERENCES}

[1] G. E. Andrews, R. A. Askey, and R. Roy, Special Functions, Cambridge University Press, Cambridge, 1999. MR1688958 (2000g:33001)

[2] C. Berg, Y. Chen, M.E.H. Ismail, Small eigenvalues of large Hankel matrices: The indeterminate case, Math. Scand. 91 (2002) 67-81. MR.1917682 (2003f:47042)

[3] Mourad E. H. Ismail, Continuous and Discrete Orthogonal Polynomials. Cambridge University Press, Cambridge, 2005.

[4] Roelof Koekoek and René F. Swarttouw, The Askey-scheme of hypergeometric orthogonal polynomials and its q-analogue, http://fa.its.tudelft.nl/ koekoek/askey/.

[5] G. Szegö, Orthogonal Polynomials, fourth edition, American Mathematics Society, Providence, RI, 1975. MR.0372517 (51:8724)

[6] Wikipedia, http://en.wikipedia.org/wiki/Hilbert_matrix

[7] J. A. Wilson, Orthogonal functions from gram determinants, SIAM J. Math. Anal. 22 (1991), 1147-1155. MR:1112071 (92c:33018)

The Institute of Applied Mathematics, College of Science, Northwest A\&F UniverSity, Yangling, ShaAnxi 712100, People's Republic of China

E-mail address: ruimingzhang@yahoo.com 